\title{
Influencia de las macrófitas sobre la estructura poblacional de rotíferos y microscrustáceos en un plano de inundación tropical
}

\author{
Silvia Lucía Villabona-González ${ }^{1}$, Néstor Jaime Aguirre R. ${ }^{1}$ \& Ana Lucía Estrada P. ${ }^{2}$ \\ 1. Grupo de investigación GAIA, Departamento de ingeniería, Universidad de Antioquia, apartado 1226, Medellín, Cra \\ 55 Av. Del Ferrocarril, Colombia; silvillabo_81@yahoo.com, naguirre@udea.edu.co \\ 2. Equipo Ambiental Proyectos, ISAGEN, Av. El Poblado Carrera 43A No 11A-80, Medellín, Colombia; \\ aestrada@isagen.com.co
}

Recibido 18-V-2010. Corregido 04-X-2010. Aceptado 02-XI-2010.

\begin{abstract}
The influence of macrophytes on rotifer and microcrustacean assemblage in a tropical floodplain. Most studies on zooplankton ecology have been conducted in open waters. However, it has been considered of great importance to extend such studies to other habitats, such as those generated of macrophytes. We studied the spatial and temporal variation of the microcrustacean and rotifer assemblage structures associated with macrophytes, and compare them with the variation exhibited in open waters. Integrated samples were collected for zooplankton and phytoplankton biomass using a Schindler bottle, in four open water sites and four other sites covered by macrophytes in the floodplain complex of Ayapel (Córdoba, Colombia) during different limnimetric levels. The significant differences in the structure were evaluated using Kruskal \& Wallis and discriminant analyses, and the similarity among sampling sites was evaluated using Bray \& Curtis analysis. Zooplanktonic richness was favored by macrophytes. However, we did not find a constant spatial pattern for density, and only particular trends apparently conditionated by flood pulses. The presence of Eichhornia azurea (Pontederiaceae) contributed in a significant way with an increase in the diversity and density of benthic taxa. The density of some zooplanktonic groups was related with environmental conditions and phytoplanktonic biomass. Rev. Biol. Trop. 59 (2): 853-870. Epub 2011 June 01.
\end{abstract}

Key words: zooplankton, floodplains, aquatic macrophytes, rotifers, cladocerans, copepods, benthos.

Uno de los grupos de organismos más característicos de los planos de inundación son las macrófitas, las cuales participan en diversos procesos del ecosistema como biomineralización y reciclaje de nutrientes. Además de esto, contribuyen significativamente en la productividad y los procesos de depuración del agua (Domínguez 2001). Se ha reportado frecuentemente que estas plantas se caracterizan por presentar complejas asociaciones con diversos grupos de organismos e influir en la distribución del zooplancton (Inger et al. 2004), otro grupo encontrado en los sistemas cenagosos, importante por su posición intermedia en la red trófica y por su capacidad indicadora de cambios ambientales (Gavilán-Díaz 2000).
El zooplancton comúnmente habita la columna de agua bajo las macrófitas y por tanto puede verse afectado por la presencia de estas plantas en un ecosistema, pues la literatura ha demostrado que las hidrófitas pueden influir sobre varias de sus características estructurales como lo son su densidad, composición, diversidad (Jeppesen et al. 1997, 1998) y distribución (Inger et al. 2004). En los planos de inundación la fluctuación de los niveles limnimétricos también es responsable de los cambios espacio-temporales en la estructura del zooplancton, debido a la alteración de las características limnológicas de los ambientes durante las diferentes fases hidrológicas (Carvalho 1983). Sin embargo, las poblaciones de 
zooplancton en estos sistemas han sido estudiadas principalmente en aguas abiertas, lo cual conlleva, en primer lugar a la subestimación de la biodiversidad local y global de este grupo, y en segundo lugar, a una carencia de postulados sobre las alteraciones que pueden producir las macrófitas en su densidad, diversidad y riqueza dentro de estos ecosistemas, sujetos a los disturbios producidos por el pulso de inundación.

En el presente estudio se pretende evaluar la variación espacial y temporal de la estructura de rotíferos y microcrustáceos asociados a las macrófitas y compararla con la variación en la estructura de rotíferos y microcrustáceos de aguas abiertas, en ocho sitios del complejo cenagoso de Ayapel, a partir de muestreos realizados en diferentes niveles limnimétricos del pulso de inundación. Así mismo, se pretende establecer el efecto de los cambios en las condiciones del ambiente sobre las variaciones espaciales y temporales en la densidad del ensamble.

\section{MATERIALES Y MÉTODOS}

Área de estudio: El complejo cenagoso de Ayapel ocupa el plano de inundación del tramo inferior de la cuenca del río San Jorge ( $8^{\circ} 04^{\prime}$ $8^{\circ} 30^{\prime} \mathrm{N}$ y $74^{\circ} 84^{\prime}-75^{\circ} 20^{\prime} \mathrm{W}$ ), ubicado en tierras bajas (22 m.s.n.m.) en la planicie atlántica del norte de Colombia, departamento de Córdoba. En general, en la zona se presenta una época de lluvias de abril a noviembre y época de sequía de diciembre a marzo. El complejo cenagoso es un sistema somero y polimíctico cálido que presenta una fluctuación pronunciada del nivel del agua a lo largo del año (Aguirre $e t$ al. 2005). Históricamente (años 1972-2000) entre febrero y marzo se registran los niveles más bajos (profundidad promedio: $2.5 \mathrm{~m}$ ) y el área mínima del espejo de agua (aproximadamente $20.6 \mathrm{~km}^{2}$ ). Luego de esto, se inicia la temporada de ascenso de las aguas que va de mayo a junio, hasta que se alcanzan el mayor nivel (profundidad promedio: $5.8 \mathrm{~m}$ ) y el área máxima (aproximadamente $140 \mathrm{~km}^{2}$ ) entre julio y noviembre. Posteriormente, entre noviembre y marzo las aguas empiezan su descenso, y de esta manera el ciclo inicia nuevamente (Wills \& Gómez 2008).

Fase de campo y laboratorio: Se eligieron ocho sitios de muestreo, cuatro en aguas abiertas (1-Ciénaga Toros, 2-Ciénaga Caimanera, 3-Ciénaga Miel, 4-Ayapel central), y cuatro bajo parches de macrófitas (5-Quebradona, 6-Ciénaga Paticos, 7-Cielo azul, 8-Caño Barro) y se realizaron campañas de muestreo entre las 7:00h y las 14:00h, en los siguientes momentos del pulso de inundación: 1. Aguas en descenso (enero 29-30 de 2008, nivel: 4.92m; febrero 3-4 de 2009, nivel: $3.77 \mathrm{~m}$ ), 2. Aguas bajas (abril 8-9 de 2008, nivel: $2.58 \mathrm{~m}$ ), 3 . Aguas en ascenso, inundación (marzo 26-27 de 2009: fase inicial de la inundación, nivel: $2.8 \mathrm{~m}$; y mayo 6-7 de 2009, nivel: $3.6 \mathrm{~m}$ ), 4. Aguas altas (julio 19-20, nivel: $6 \mathrm{~m}$ y septiembre $12-13$ de 2008, nivel: $5.8 \mathrm{~m})$.

El zooplancton fue recolectado con una botella Schindler de 5L, integrando diferentes profundidades equidistantes dentro de la columna de agua (de acuerdo con la profundidad del sitio de muestreo) hasta completar un volumen de $35 \mathrm{~L}$. Las muestras fueron concentradas con un tamiz de $65 \mu \mathrm{m}$, almacenadas en frascos de $50 \mathrm{ml}$ y fijadas con solución transeau (seis partes de agua, tres de alcohol y una de formol) en proporción 1:1. Se contaron cuántos mililitros fueron necesarios (en alícuotas de $1 \mathrm{ml}$ ) para alcanzar 100 individuos de cada uno de los dos taxones de zooplancton más abundantes (Estrada-Posada 2007). Las muestras donde las densidades no superaron los 100 organismos del taxón más abundante fueron contadas en su totalidad y en los casos donde se presentaba demasiado sedimento, se contaron $10 \mathrm{ml}$ de muestra en alícuotas de $1 \mathrm{ml}$.

La determinación taxonómica se realizó usando las siguientes claves taxonómicas: Rotifera (Koste 1978, Koste \& Shield 1987, Shield \& Koste 1992, 1993, Segers 1995, Fernando 2002), Cladocera (Paggi 1995, Alonso 1996, El Moor-Loureiro 1997, Elías et al. 2008) y Copepoda (Sendacz \& Kubo 1982, Reid 1985, Suárez et al. 1996, Gaviria 2000). El hábito de los taxones se documentó de: 
Wallace \& Edmonson (1986), Bonecker et al. (1998), Rossa \& Bonecker (2003) y Gaviria \& Aranguren (2007).

De la misma forma que para el zooplancton, se tomaron muestras integradas de la columna de agua para evaluar la biomasa del fitoplancton, que se estimó mediante el método de la clorofila $a$ (Clesceri et al. 1998), la muestra de agua se pasó a través de un filtro de fibra de vidrio Whatman $(0.45 \mu \mathrm{m}$ de poro y $47 \mathrm{~mm}$ de diámetro) y como solvente extractor se utilizó etanol caliente al 96\%. Para discriminar entre clorofila $a$ y feopigmentos, se acidificó el extracto con $\mathrm{HCl}$ (Sartory \& Grobbelaar 1984).

En cada uno de los sitios cubiertos por macrófitas se lanzó un cuadrante de $1 \mathrm{~m}^{2}$ ( $\sin$ sub-cuadrantes) dentro del cual se estimó el porcentaje de cobertura de cada especie de macrófita. Las coberturas obtenidas se convirtieron a la escala de importancia de DominKrajina (Thomaz et al. 2004).

Se calculó la riqueza numérica de especies, la diversidad específica (Shannon \& Weaver 1949), la equidad (Pielou 1969) y la dominancia (Simpson 1949). El número de especies esperado fue estimado mediante los estimadores Chao 2 y Jacknife 1 y se empleó un análisis de similaridad de Bray-Curtis con ligamiento simple, para analizar la similitud espacial con base en la presencia/ausencia del zooplancton. Estos análisis se ejecutaron en el programa Biodiversity Pro ver 2.0, se empleó un análisis de discriminantes, ejecutado en el Programa SPSS ver 13.0 para determinar la existencia de patrones espaciales y temporales en función de la densidad de 41 taxones de zooplancton. Para escoger dichos taxones y descartar los taxones raros (que aportaron poco a la varianza) se empleó un análisis exploratorio de componentes principales en el programa STATISTICA 6.0. Se utilizó la prueba no paramétrica de Kruskal \& Wallis con el fin de evidenciar diferencias de la densidad total del zooplancton y de los índices de diversidad entre los sitios y temporadas de muestreo, y para evidenciar relaciones entre variables se aplicó el coeficiente de correlación de Spearman y la regresión lineal simple. Para lo anterior se empleó el programa STATISTICA 6.0. Los datos de las variables físicas y químicas para las correlaciones fueron tomados del análisis del componente abiótico realizado simultáneamente (Villabona-González 2010).

\section{RESULTADOS}

Composición del zooplancton: El zooplancton estuvo conformado por 125 taxones, que incluyeron nauplios y copepoditos de los tres órdenes de Copepoda hallados. De acuerdo a los estimadores la riqueza observada estuvo muy cerca de la esperada (Chao $2=136$ taxones; Jacknife 1=139 taxones). Los rotíferos fueron el grupo más rico en taxones con un $62 \%$ de la riqueza total (75 taxones), seguido de los microcrustáceos, dentro de los cuales los cladóceros contribuyeron con un $24.8 \%$ de la riqueza (29 taxones) y los copépodos con un 7.4\% (9 taxones). Además, se registraron cinco taxones de protozoos $(4.1 \%)$ y tres de ostrácodos (2.5\%), aunque esta información es incompleta por la dificultad de preservación e identificación de dichos grupos. La riqueza taxonómica dentro de las aguas abiertas fue de 91 taxones y de estos, los rotíferos Gastropus sp. y Horaella cf thomassoni Koste y los cladóceros Euryalona sp. e Ilyocryptus spinosus Štifter se encontraron sólo en dicho hábitat. El hábitat de las macrófitas tuvo una riqueza total de 118 taxones, 30 de ellos exclusivos (20 rotíferos, ocho cladóceros y dos protozoos) (Cuadro 1). El análisis de Bray \& Curtis muestra una asociación entre los sitios influenciados por la presencia de las macrófitas (con excepción de la ciénaga Paticos), con porcentajes de similaridad mayores al 80\%. De igual forma, los sitios ubicados en aguas abiertas fueron muy similares entre sí, mostrando índices de similaridad mayores al 70\%; dentro de éstos, las ciénagas Miel y Toros se agruparon con un porcentaje del $79.1 \%$ y la ciénaga Caimanera y el sector Ayapel central con un porcentaje de $78.2 \%$ (Fig. 1). Dentro de los sitios de aguas abiertas estuvieron mejor representadas las especies euplanctónicas $(59.3 \%)$ y por lo tanto, las formas bentónicas estuvieron en menor cantidad 
CUADRO 1

Composición taxonómica y hábito predominante del zooplancton. P: planctónico; B: bentónico

TABLE 1

Taxonomic composition and dominant habit of zooplankton. P: planktonic; B: benthic

\begin{tabular}{|c|c|c|c|c|c|}
\hline Phylum & Clase & Orden & Familia & Taxón & Hábito \\
\hline \multirow[t]{33}{*}{ Rotifera } & Bdelloidea & Bdelloidea & & Bdelloideo & B \\
\hline & Monogononta & Collothecaceae & Collothecidae & Collotheca sp. 1 & B \\
\hline & & & & Collotheca sp. $2^{*}$ & B \\
\hline & & & & Collotheca sp. 3 & B \\
\hline & & & & Collotheca sp. 4 & B \\
\hline & & & & Collotheca sp. $5^{*}$ & $\mathrm{~B}$ \\
\hline & & Floscularicea & Conochilidae & Conochilus dossuarius Hudson, 1885 & $\mathrm{P}$ \\
\hline & & & & Conochilus natans (Seligo,1900) & $\mathrm{P}$ \\
\hline & & & Filiniidae & Filinia longiseta (Ehrenberg, 1834) & $\mathrm{P}$ \\
\hline & & & & Filinia opoliensis (Zacharias, 1898) & $\mathrm{P}$ \\
\hline & & & & Filinia pejleri Hutchinson,1964 & $\mathrm{P}$ \\
\hline & & & & Filinia terminalis (Plate, 1886) & $P$ \\
\hline & & & Hexarthridae & Hexarthra mira (Hudson,1871) & $\mathrm{P}$ \\
\hline & & & Testudinellidae & Testudinella mucronata (Gosse,1886) & $\mathrm{P}$ \\
\hline & & & & Testudinella patina (Hermann, 1783) & $\mathrm{B}$ \\
\hline & & & Trochosphaeridae & Horaella cf thomassoni + Koste,1973 & $P$ \\
\hline & & Ploima & Asplanchnidae & Asplanchna sieboldii (Leydig, 1854) & $\mathrm{P}$ \\
\hline & & & Brachionidae & Brachionus angularis Gosse, 1851 & $\mathrm{P}$ \\
\hline & & & & Brachionus bidentatus Anderson, 1889 & $\mathrm{P}$ \\
\hline & & & & Brachionus budapestinensis Daday, 1885 & $\mathrm{P}$ \\
\hline & & & & Brachionus calyciflorus Pallas, 1776 & $\mathrm{P}$ \\
\hline & & & & Brachionus caudatus Barrois \& Daday, 1894 & $\mathrm{~B}$ \\
\hline & & & & Brachionus dimidiatus * Bryce, 1931. & $\mathrm{P}$ \\
\hline & & & & Brachionus diversicornis (Daday,1883) & $\mathrm{P}$ \\
\hline & & & & Brachionus dolabratus Harring, 1914 & $P$ \\
\hline & & & & Brachionius falcatus Zacharias, 1898 & $P$ \\
\hline & & & & Brachionus havanaensis Rousselet, 1911 & $\mathrm{P}$ \\
\hline & & & & Brachionus mirus Daday, 1905 & $\mathrm{P}$ \\
\hline & & & & $\begin{array}{l}\text { Brachionus quadridentatus "f". mirabilis Daday,1897 y } \\
\text { "f". quadridentatus Hermann, } 1783\end{array}$ & $\mathrm{P}$ \\
\hline & & & & Keratella americana Carlin, 1943 & $\mathrm{P}$ \\
\hline & & & & Keratella cochlearis (Gosse, 1851) & $\mathrm{P}$ \\
\hline & & & & Keratella lenzi Hauer, 1953 & $\mathrm{P}$ \\
\hline & & & & Keratella tropica (Apstein,1907) & $\mathrm{P}$ \\
\hline
\end{tabular}


CUADRO 1 (Continuación)

Composición taxonómica y hábito predominante del zooplancton. P: planctónico; B: bentónico

TABLE 1 (Continued)

Taxonomic composition and dominant habit of zooplankton. P: planktonic; B: benthic

\begin{tabular}{|c|c|c|c|c|c|}
\hline Phylum & Clase & Orden & Familia & Taxón & Hábito \\
\hline & & & & $\begin{array}{l}\text { Plationus patulus "f". macracanthus (Daday, 1905) y "f". } \\
\text { patulus (O. F. Müller,1786) }\end{array}$ & $\mathrm{P}$ \\
\hline & & & & Platyias leloupi Gillard, 1967 & $\mathrm{P}$ \\
\hline & & & & Platyias quadricornis (Ehrenberg, 1832) & $P$ \\
\hline & & & Epiphanidae & Epiphanes macroura (Barrois \& Daday, 1894) & B \\
\hline & & & Euchlanidae & Euchlanis sp. & \\
\hline & & & Gastropodidae & Ascomorpha sp. & \\
\hline & & & & Gastropus sp. + & B \\
\hline & & & Lecanidae & Lecane bulla (Gosse, 1851) & B \\
\hline & & & & Lecane cornuta (O. F. Müller,1786) & B \\
\hline & & & & Lecane curvicornis (Murray, 1913) & B \\
\hline & & & & Lecane elsa Hauer,1931 & B \\
\hline & & & & Lecane cf hastata* (Murray, 1913) & B \\
\hline & & & & Lecane cf hornemanni* (Ehrenberg, 1834) & B \\
\hline & & & & Lecane leontina (Turner,1892) & B \\
\hline & & & & Lecane ludwigii " $\mathrm{f}$ ". ercodes* (Eckstein, 1883) & B \\
\hline & & & & Lecane luna (Müller,1776) & B \\
\hline & & & & Lecane lunaris* (Ehrenberg, 1832) & B \\
\hline & & & & Lecane papuana (Murray, 1913) & B \\
\hline & & & & Lecane proiecta Hauer, 1956 & B \\
\hline & & & & Lecane pumila* (Rousselet, 1906). & B \\
\hline & & & & Lecane pyriformis* (Daday,1905) & B \\
\hline & & & & Lecane quadridentata* $($ Ehrenberg, 1830$)$ & B \\
\hline & & & & Lecane signifera* (Jennings,1896) & B \\
\hline & & & & Lecane sp.* & B \\
\hline & & & Lepadellidae & Lepadella cf amazonica Segers, 1993 & B \\
\hline & & & & 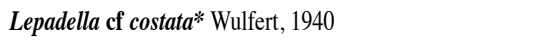 & B \\
\hline & & & & Lepadella cf patella* (Müller, 1773) & B \\
\hline & & & Mytilinidae & Mytilina ventralis* (Ehrenberg, 1830) & B \\
\hline & & & Scaridiidae & Scaridium sp.* & \\
\hline & & & Synchaetidae & Ploesoma truncatum (Levander, 1894) & $\mathrm{P}$ \\
\hline & & & & Polyarthra dolichoptera Idelson,1925 & $\mathrm{P}$ \\
\hline & & & & Synchaeta stylata Wierzejski, 1893 & $\mathrm{P}$ \\
\hline & & & Trichocercidae & Trichocerca agnatha Wulfert, 1939 & $\mathrm{P}$ \\
\hline & & & & Trichocerca bicristata (Gosse, 1887) & $\mathrm{P}$ \\
\hline
\end{tabular}


CUADRO 1 (Continuación)

Composición taxonómica y hábito predominante del zooplancton. P: planctónico; B: bentónico

TABLE 1 (Continued)

Taxonomic composition and dominant habit of zooplankton. P: planktonic; B: benthic

\begin{tabular}{|c|c|c|c|c|c|}
\hline Phylum & Clase & Orden & Familia & Taxón & Hábito \\
\hline & & & & Trichocerca cf capucina* (Wierzejski \& Zacharias, 1893) & $\mathrm{P}$ \\
\hline & & & & Trichocerca chattoni (De Beauchamp, 1907) & $\mathrm{P}$ \\
\hline & & & & Trichocerca cf pusilla (Jennings, 1903) & $\mathrm{P}$ \\
\hline & & & & Trichocerca similis (Wierzejski, 1893) & $P$ \\
\hline & & & & Trichocerca cf stylata* (Gosse,1851) & $P$ \\
\hline & & & & Trichocerca sp.* & \\
\hline & & & Trichotriidae & Macrochaetus sp.* & \\
\hline & & & & Trichotria tetractis (Ehrenberg, 1830) & B \\
\hline \multirow[t]{26}{*}{ Arthropoda } & Branchiopoda & Anomopoda & Bosminidae & Bosmina tubicen Brehm, 1953 & $P$ \\
\hline & & & & Bosmina longirostris (O. F. Müller,1785) & $P$ \\
\hline & & & & Bosminopsis deitersi Richard, 1895 & $P$ \\
\hline & & & Chydoridae & Alona guttata* Sars, 1862 & B \\
\hline & & & & Alona sp. 1 & B \\
\hline & & & & Alona sp. 2 & $\mathrm{~B}$ \\
\hline & & & & Alona sp. 3* & $\mathrm{B}$ \\
\hline & & & & Alonella sp. & $\mathrm{B}$ \\
\hline & & & & Camptocercus dadayi Stingelin, 1913 & B \\
\hline & & & & Chydorus sp. 1 & $\mathrm{~B}$ \\
\hline & & & & Chydorus sp. $2 *$ & $\mathrm{~B}$ \\
\hline & & & & Euryalona sp.+ & $\mathrm{B}$ \\
\hline & & & & Leydigia acanthocercoides (Fischer, 1854) & $\mathrm{B}$ \\
\hline & & & & Notoalona cf sculpta* (Sars, 1901) & $\mathrm{B}$ \\
\hline & & & Daphniidae & Ceriodaphnia cornuta s. 1. Sars, 1886 & $\mathrm{P}$ \\
\hline & & & & Ceriodaphnia rigaudi s.l. Sars, 1886 & $\mathrm{P}$ \\
\hline & & & & Simocephalus cf expinosus (Koch, 1841) & $\mathrm{P}$ \\
\hline & & & Ilyocryptidae & Ilyocryptus spinifer* Herrick, 1884 & $\mathrm{~B}$ \\
\hline & & & & Ilyocryptus spinosus + Štifter, 1988 & $\mathrm{~B}$ \\
\hline & & & Macrothricidae & Macrothrix sp. & $\mathrm{B}$ \\
\hline & & & Moinidae & Moina minuta Hansen, 1891 & $\mathrm{P}$ \\
\hline & & & & Moina micrura (Kurz, 1874) & $\mathrm{P}$ \\
\hline & & & & Moina reticulata (Daday, 1905) & $\mathrm{B}$ \\
\hline & & Ctenopoda & Sididae & Diaphanosoma birgei Korínek, 1981 & $\mathrm{P}$ \\
\hline & & & & Diaphanosoma brevireme*, Sars,1901 & $\mathrm{P}$ \\
\hline & & & & Diaphanosoma dentatum Herbst, 1968 & $\mathrm{P}$ \\
\hline
\end{tabular}


CUADRO 1 (Continuación)

Composición taxonómica y hábito predominante del zooplancton. P: planctónico; B: bentónico

TABLE 1 (Continued)

Taxonomic composition and dominant habit of zooplankton. P: planktonic; B: benthic

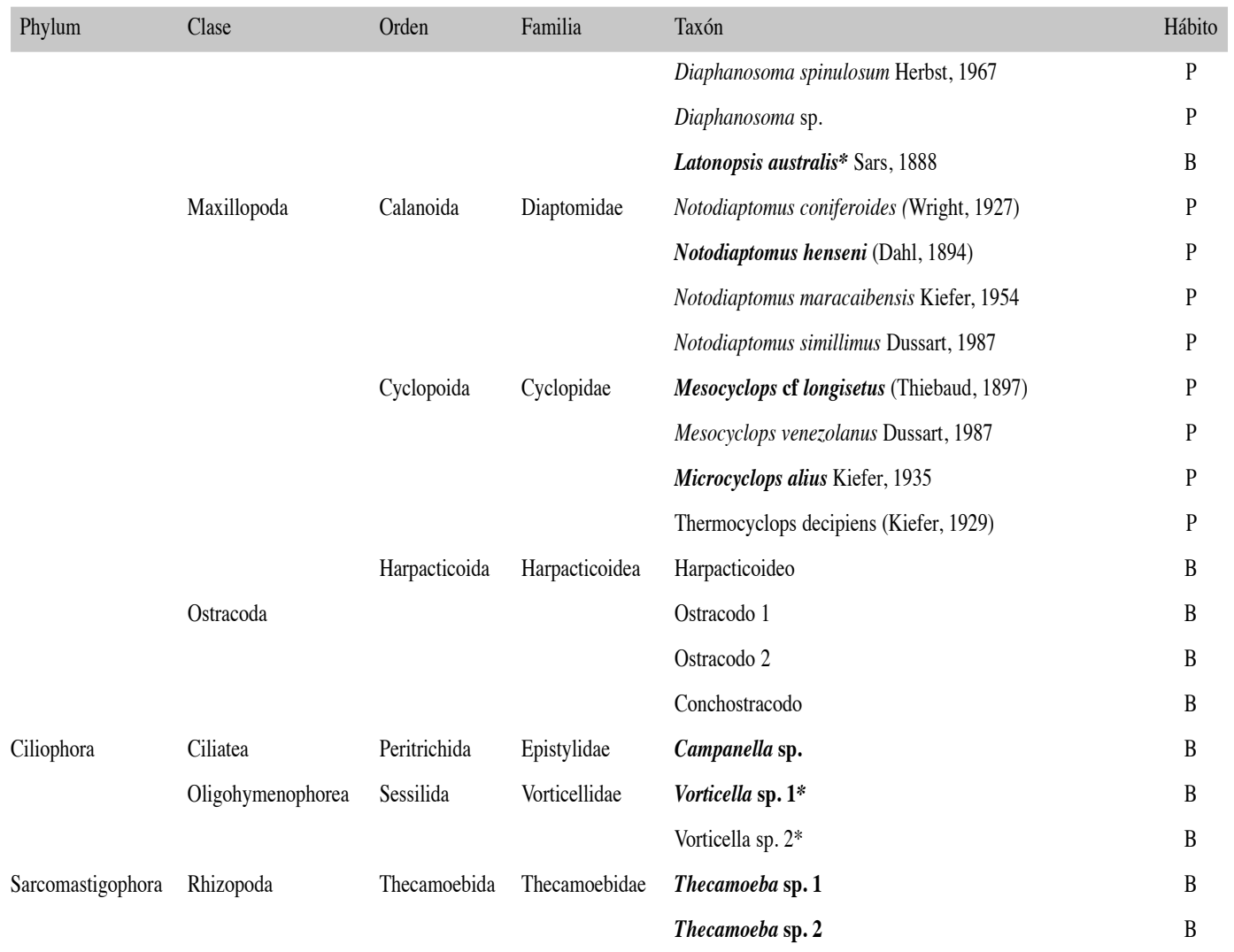

En negrilla primer reporte para el área de estudio.

* Restringido al hábitat de las macrófitas.

+ Restringido a las aguas abiertas.

(40.6\%), aunque también fueron abundantes. Bajo las macrófitas, la proporción de formas no planctónicas fue mayor que en las aguas abiertas y de esta manera la mitad $(50 \%)$ de los individuos registrados fueron organismos típicamente litorales (Cuadro 1).

Densidad del zooplancton: La densidad total del zooplancton y la densidad de cada uno de los grupos de zooplancton (rotíferos, cladóceros, copépodos, protozoos y ostrácodos) no presentaron diferencias significativas entre los sitios analizados. En la escala temporal la densidad del zooplancton se relacionó negativamente con el nivel de las aguas $(\mathrm{R}=-0.78$, $\mathrm{p}<0.05$ ), de esta manera en los niveles más bajos hubo un mayor promedio de individuos por unidad de volumen, 8.2ind./L en abril y 6.6ind./ L en marzo; y en julio, cuando se registró el mayor nivel en el complejo cenagoso, se registró la menor densidad (media 3.0ind./L). A pesar de esto, el test de Kruskal \& Wallis agrupado por las siete temporadas de muestreo, no arrojó diferencias significativas. Así mismo, al 


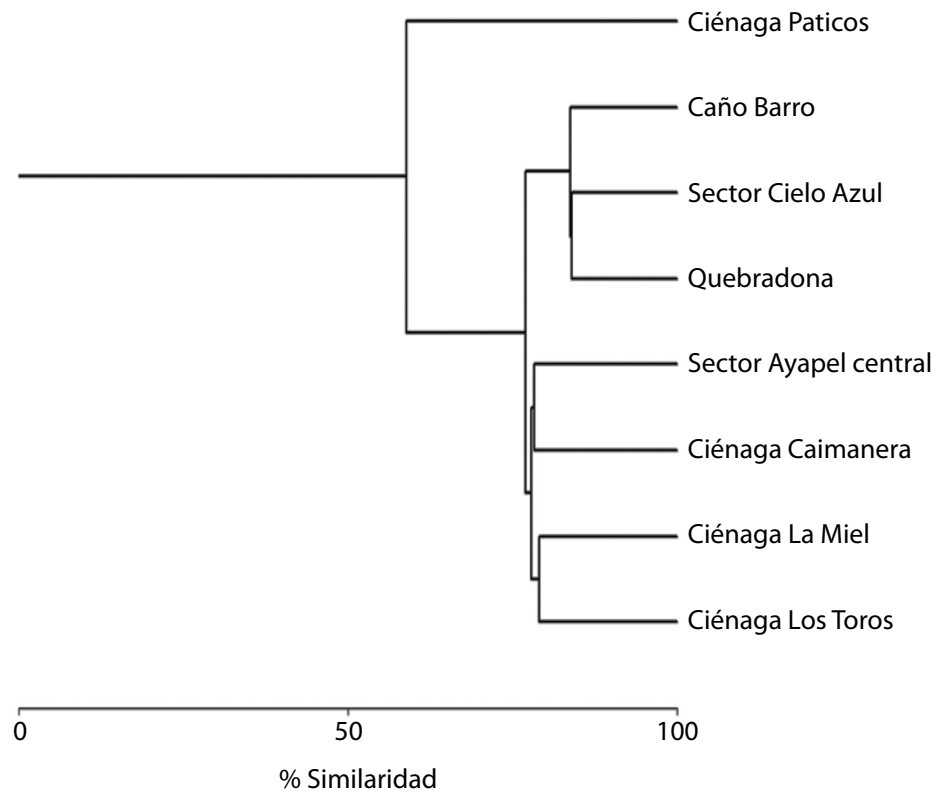

Fig. 1. Análisis de similaridad para el zooplancton en el complejo cenagoso de Ayapel. Fig. 1. Similarity analysis of zooplankton in the Ayapel floodplain complex.

analizar por separado la densidad de cada uno de los cinco grupos de zooplancton, no se registraron diferencias temporales significativas.

A pesar de la ausencia de cambios significativos estadísticamente en espacio y tiempo, se identifican algunas tendencias como la disminución de la densidad en los sitios de aguas abiertas ubicados en el extremo norte del complejo cenagoso durante la inundación de marzo y mayo de 2009 y el mayor número de individuos, en especial de cladóceros, dentro de los sitios cubiertos por las plantas durante estas mismas temporadas. También se observa que generalmente los protozoos y ostrácodos fueron más numerosos en los sitios cubiertos por macrófitas y que en la ciénaga La Miel el zooplancton fue muy abundante (Fig. 2).

Los nauplios (media 78.2ind./L) y copepoditos de Cyclopoida (media 50.6ind./L) fueron en general las formas más abundantes. Dentro de los rotíferos, Brachionus calyciflorus Pallas (20.2ind./L) y Brachionus caudatus Barrois \& Daday (11.7ind./L) alcanzaron la mayor densidad media y dentro de los cladóceros Moina minuta Hansen (media 11ind./L) y Diaphanosoma birgei Korínek (media 4.5ind./L) fueron los más abundantes. Notodiaptomus maracaibensis Kiefer (media 3.18ind./L) fue el copépodo más numeroso dentro de las aguas abiertas y Thermocyclops decipiens Kiefer (media 3.41ind./L), el más numeroso dentro de las camas de macrófitas. Adicionalmente, el cladócero Moina reticulata Daday y los rotíferos Bdelloideo, Brachionus bidentatus Anderson, Ploeosoma truncatum Levander y Ascomorpha sp., presentaron altas densidades medias dentro de las plantas pero no en las aguas abiertas y los rotíferos Lecane proiecta Hauer y $\mathrm{Bra}$ chionus havanaensis Rousselet presentaron el comportamiento contrario.

Análisis discriminante: En el análisis agrupado por sitios, se obtuvo un porcentaje de clasificación de $94.6 \%$ y sólo la primera función fue significativa (Lambda-Wilks, $\mathrm{p}<0.05$ ), 


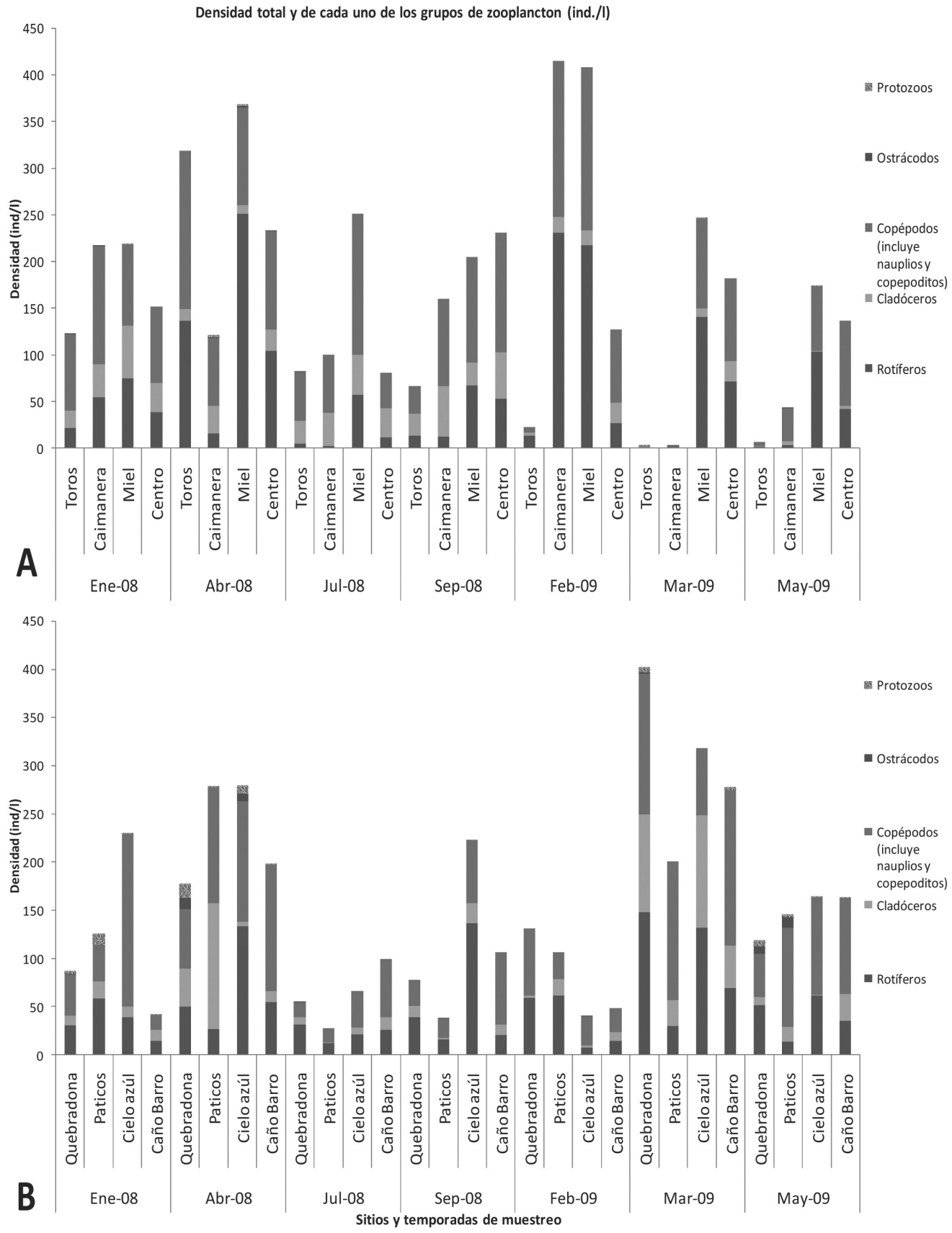

Fig. 2. Densidad total y de cada uno de los grupos del zooplancton en el complejo cenagoso de Ayapel. A. Aguas abiertas. B. Macrófitas.

Fig. 2. Total density and density of each zooplankton group in the Ayapel floodplain complex. A. Open water. B. Macrophytes. 
indicando una varianza de $63.7 \%$. El análisis revela que dos sitios limnéticos: Ciénaga La Miel (3) y Ayapel central (4) y un sitio bajo las macrófitas, Quebradona (5) presentan diferencias significativas; dichos lugares difieren por la presencia de altas densidades de los rotíferos Conochilus dossuarius Hudson, Asplanchna sieboldii Leydig y Epiphanes macroura Barrois \& Daday, en el caso de los sitios limnéticos y por las mayores densidades de organismos típicamente litorales como Macrothrix sp., Lecane leontina Turner y Alona sp. 2 en el caso del sitio bajo las plantas (Fig. 3). El análisis agrupado por temporadas tiene un porcentaje de clasificación del 100\% y dos funciones significativas (Lambda-Wilks: Función 1 y Función 2: p<0.05). El análisis muestra que las temporadas de aguas en descenso de enero de 2008 y de aguas bajas de abril de 2008 son diferentes de las demás. Esto es gracias a que taxones como Brachionus havanaensis, Thecamoeba sp.1, Keratella tropica Apstein y Diaphanosoma sp. fueron muy abundantes sólo en ambas temporadas, sobre todo en la época de menor nivel y a que cladóceros como Ceriodaphnia rigaudi Sars, Ceriodaphnia cornuta Sars y Bosminopsis deitersi Richard fueron abundantes en las otras cinco temporadas y no en enero y abril de 2008 (función 1, varianza: $77.6 \%$ ). Las aguas en descenso y las aguas bajas difieren entre sí, ya que en enero hubo una alta densidad de Conochilus natans Seligo, Conochilus dossuarius y Brachionus falcatus Zacharias y en abril de Brachionus calyciflorus, Epiphanes macroura y Trichocerca similis Wierzejski. Estos rotíferos, al igual que los cladóceros anteriormente mencionados, fueron muy abundantes en las épocas de inundación de marzo y mayo de 2009, haciendo que los periodos de ingreso de los tributarios fueran similares entre sí y diferentes de los demás (función 2, varianza: 10.9\%) (Fig. 4).

Descriptores de diversidad: Generalmente dentro de las macrófitas, el zooplancton tuvo una mayor riqueza taxonómica que en las aguas abiertas, lo cual fue estadísticamente significativo (Kruskal \& Wallis, $\mathrm{p}<0.05$ ) al tener en

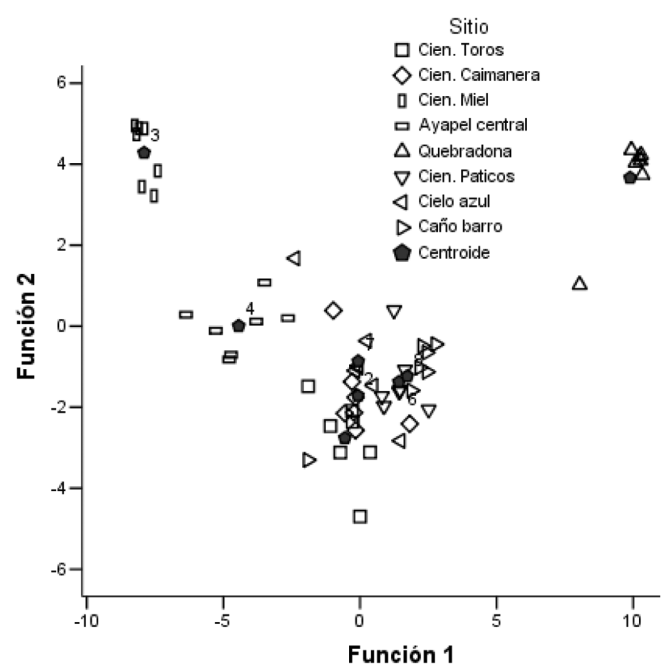

Fig. 3. Análisis discriminante del zooplancton agrupado por sitios de muestreo.

Fig. 3. Discriminant analysis of zooplankton grouped by sampling sites.

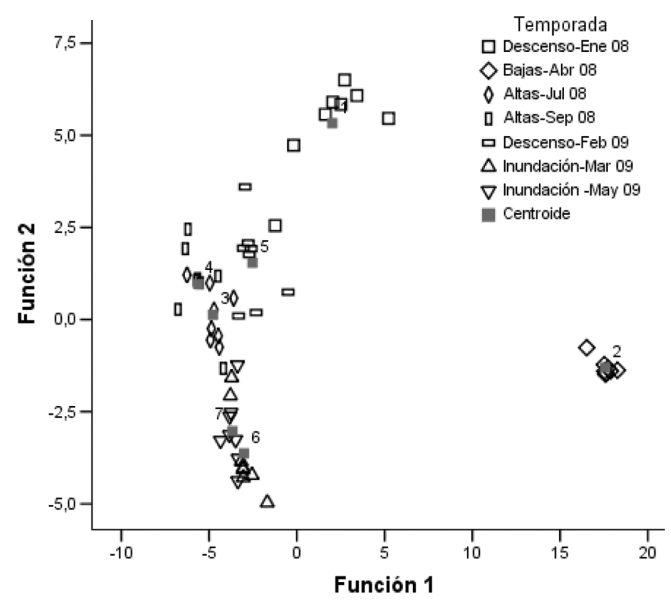

Fig. 4. Análisis discriminante del zooplancton agrupado por temporadas de muestreo.

Fig. 4. Discriminant analysis of zooplankton grouped by season of sampling.

cuenta todos los muestreos. Temporalmente, la riqueza no mostró una variación significativa, ni tomando en cuenta todos los sitios, ni analizando por separado los dos hábitats. El índice de Shannon varió entre 1.18nat/ind. y 2.77nat/ 
ind. Las diferencias en el espacio (Kruskal \& Wallis $\mathrm{p}<0.05)$, estuvieron determinadas porque generalmente se presentaron diversidades mayores en Quebradona, dominado por Eichhornia azurea (Sw.) Kunth. En la escala temporal el ensamble no presentó variaciones estadísticas significativas al tener en cuenta todos los sitios de muestreo. La equidad fue alta en la mayoría de los casos $(>0.5)$ y la dominancia presentó valores bajos $(<0.5)$, siendo en todos los casos menor que la equidad. Ninguno de estos dos descriptores presentó diferencias significativas en espacio y tiempo (Fig. 5).

Asociación del zooplancton con las variables físicas, químicas y biológicas: Las correlaciones significativas entre las densidades del zooplancton (densidad total, densidad de cada uno de los grupos zoológicos y densidad por taxón) y las variables analizadas tuvieron valores bajos de asociación $(<0.5)$ en la mayoría de los casos, a excepción de los

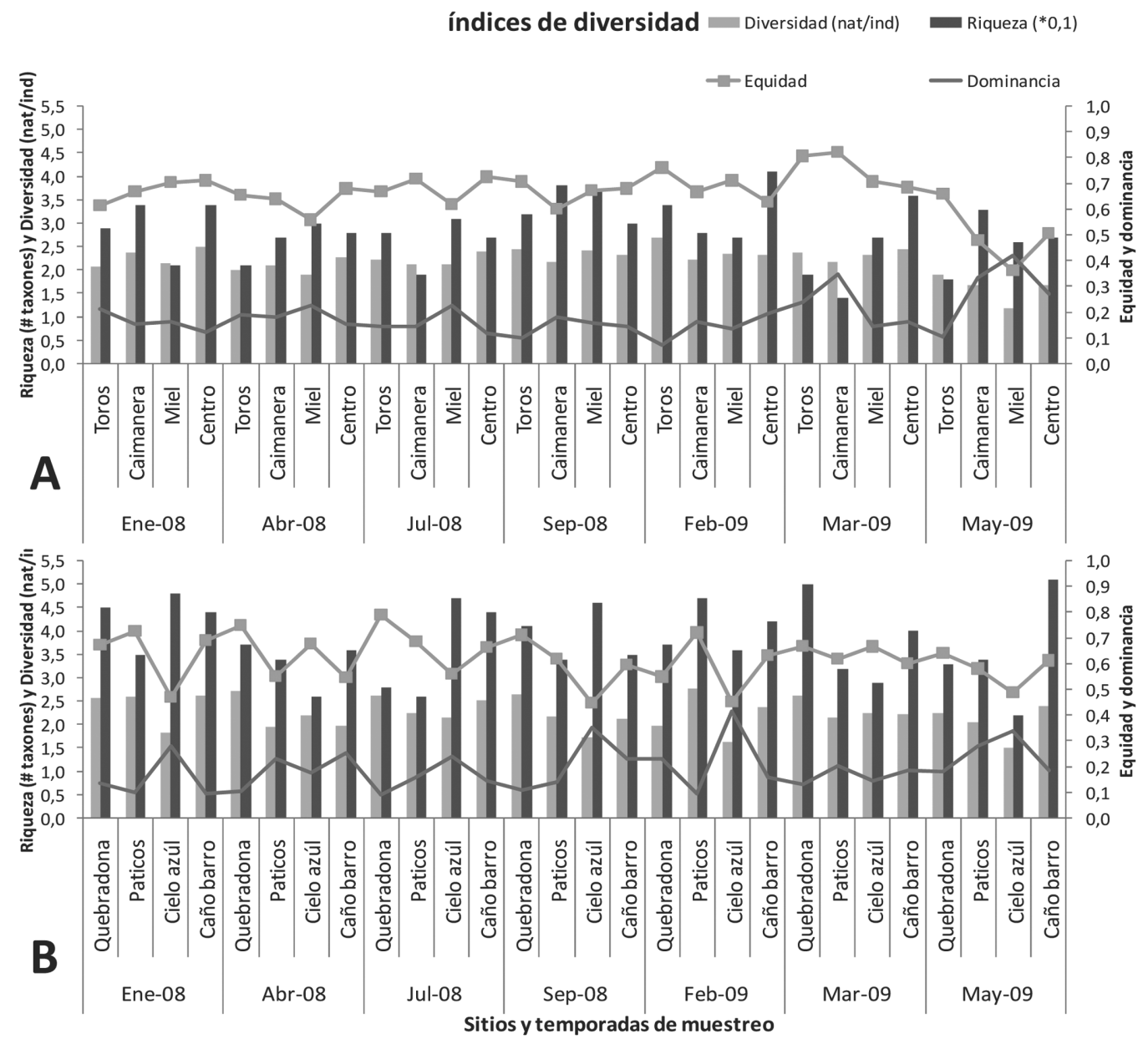

Fig. 5. Variación espacial y temporal de los valores de diversidad $\left(\mathrm{H}^{\prime}\right)$, Equidad $\left(\mathrm{J}^{\prime}\right)$ y dominancia (D) del zooplancton. A. Aguas abiertas. B. Macrófitas.

Fig. 5. Spatial and temporal variation of the diversity (H '), evenness (J') and dominance (D) values of zooplankton. A. Open waters. B. Macrophytes. 
rotíferos Lecane proiecta, que se relacionó positivamente con el oxígeno disuelto $(\mathrm{R}=0.5$, $\mathrm{p}<0.05)$ y Conochilus dossuarius, que presentó una correlación negativa con la concentración de sólidos suspendidos $(\mathrm{R}=-0.55, \mathrm{p}<0.05)$. Las correlaciones realizadas para cada hábitat (densidad total y de cada grupo) mostraron que en las aguas abiertas la mayor densidad de rotíferos se asoció con altos valores de oxígeno disuelto y biomasa de fitoplancton y que los cladóceros se asociaron de forma negativa con los nutrientes y la concentración de sólidos totales y suspendidos (Cuadro 2). Dentro de las plantas no hubo correlaciones significativas.

\section{CUADRO 2}

Correlaciones significativas $(\mathrm{p}<0.05)$ entre el zooplancton y el componente abiótico y la clorofila a

TABLE 2

Significant correlation $(\mathrm{p}<0.05)$ between zooplankton and abiotic component and chlorophyll a

\begin{tabular}{lcc}
\multicolumn{1}{c}{ Correlación } & $\mathrm{R}$ & $\mathrm{p}$ \\
Rotíferos \& oxígeno disuelto & 0.63 & 0.000278 \\
Rotíferos \& clorofila $a$ & 0.5 & 0.006248 \\
Cladóceros \& amonio & -0.58 & 0.001027 \\
Cladóceros \& nitratos & -0.71 & 0.00002 \\
Cladóceros \& ortofosfatos & -0.67 & 0.000078 \\
Cladóceros \& sólidos totales & -0.62 & 0.000389 \\
Cladóceros \& sólidos suspendidos & -0.5 & 0.004478
\end{tabular}

Como se observa en el Cuadro 3, las camas de macrófitas que cubrían los cuatro sitios seleccionados estuvieron conformadas por Eichhornia crassipes (Mart.) Solms, Eichhornia heterosperma Alexander, Eichhornia azurea y Oxycaryum cubense (Poepp. \& Kunth) Palla. En el cuadro también se evidencia que fueron E. crassipes y E. heterosperma las plantas que con mayor frecuencia formaron estos parches y que a su vez fueron las que generalmente más aportaron a la cobertura total, de acuerdo con la escala de importancia de cobertura de Domin Krajina. La densidad total de zooplancton no estuvo explicada por el porcentaje de cobertura de las plantas, a pesar de que la cobertura de algunas especies encontradas varió significativamente en espacio y tiempo, como es el caso de E. crassipes que presentó la cobertura máxima (valor de importancia de 5 en todos los sitios de muestreo) durante el ingreso de los tributarios en marzo (Kruskal $\&$ Wallis $\mathrm{p}<0.05)$ y de E. azurea que tuvo una mayor cobertura en Quebradona (Kruskal \& Wallis $\mathrm{p}<0.05)$ y se correlacionó positivamente con la densidad de Macrothrix sp. ( $\mathrm{R}=0.51$, $\mathrm{p}<0.05)$, Lecane leontina $(\mathrm{R}=0.45, \mathrm{p}<0.05)$ y Alona sp. $2(\mathrm{R}=0.55, \mathrm{p}<0.05)$.

\section{DISCUSIÓN}

Con base en el cálculo de los estimadores de riqueza y la construcción de la curva de acumulación de taxones, se considera que la cantidad de muestreos realizados es fiable. Se registraron 75 taxones de rotíferos, 29 de cladóceros, nueve de copépodos, cinco de protozoos y tres de ostrácodos. La mayor riqueza de rotíferos en planos de inundación ha sido registrada por Aranguren (1998), Gavilán-Díaz (2000) y Andrade (2001), entre otros y específicamente en el complejo cenagoso de Ayapel en las investigaciones de Palacio (2008) y Gallo-Sánchez et al. (2009). Brachionus caudatus y B. calyciflorus fueron los rotíferos más numerosos. Andrade (2001) encontró que $B$. caudatus se relaciona con una alta disponibilidad de material orgánico particulado y de la biota encargada de su descomposición (hongos y bacterias), lo que se esperaría ocurriera en un sistema como Ayapel donde las altas temperaturas y la resuspensión del sedimento debido a la baja profundidad y fuerza del viento favorecerían altas tasas de descomposición de materia orgánica (Aguirre et al.2005). B calyciflorus es una especie cosmopolita que tolera altos grados de contaminación y es considerada indicadora de ambientes mesosaprobios (Sladecek 1983).

Los cladóceros presentaron una mayor diversidad de formas bentónicas (Familia Chydoridae), lo cual está altamente influenciado por la exploración de la columna de agua bajo las macrófitas y por el arrastre accidental hacia la zona pelágica. Rossa et al. (2001) 
CUADRO 3

Valores de importancia del porcentaje de cobertura y frecuencia absoluta de las macrófitas

TABLE 3

Importance values of percentage of coverage and absolute frequency of macrophytes

\begin{tabular}{|c|c|c|c|c|c|}
\hline \multirow[b]{2}{*}{ Temporada } & \multirow[b]{2}{*}{ Sitio } & \multicolumn{4}{|c|}{ Valor de importancia para la cobertura } \\
\hline & & $\begin{array}{l}\text { Eichhornia } \\
\text { crassipes }\end{array}$ & $\begin{array}{c}\text { Eichhornia } \\
\text { heterosperma }\end{array}$ & $\begin{array}{c}\text { Eichhornia } \\
\text { azurea }\end{array}$ & $\begin{array}{l}\text { Oxycarium } \\
\text { cubense }\end{array}$ \\
\hline \multirow{4}{*}{ Ene-08 } & Quebradona & 1 & 3 & & 2 \\
\hline & Ciénaga Paticos & 4 & 2 & & \\
\hline & Cielo azul & 4 & 1 & & 1 \\
\hline & Caño Barro & 3 & 3 & & \\
\hline \multirow{4}{*}{ Abr-08 } & Quebradona & & & 5 & \\
\hline & Ciénaga Paticos & & & 5 & \\
\hline & Cielo azul & 5 & & & 1 \\
\hline & Caño Barro & & 5 & & \\
\hline \multirow{4}{*}{ Jul-08 } & Quebradona & & & 5 & \\
\hline & Ciénaga Paticos & 5 & 1 & & \\
\hline & Cielo azul & 4 & 2 & & \\
\hline & Caño Barro & 5 & 1 & & \\
\hline \multirow{4}{*}{ Sep-08 } & Quebradona & & & 5 & \\
\hline & Ciénaga Paticos & 1 & 5 & & \\
\hline & Cielo azul & 1 & 5 & & \\
\hline & Caño Barro & & 5 & & \\
\hline \multirow{4}{*}{ Feb-09 } & Quebradona & 2 & 4 & & \\
\hline & Ciénaga Paticos & 2 & & 5 & \\
\hline & Cielo azul & & 2 & 3 & \\
\hline & Caño Barro & 4 & 2 & & \\
\hline \multirow{4}{*}{ Mar-09 } & Quebradona & 5 & & & \\
\hline & Ciénaga Paticos & 5 & & 1 & \\
\hline & Cielo azul & 5 & & & \\
\hline & Caño Barro & 5 & & & \\
\hline \multirow{4}{*}{ May-09 } & Quebradona & & & 5 & \\
\hline & Ciénaga Paticos & 1 & & 4 & \\
\hline & Cielo azul & & 5 & & \\
\hline & Caño Barro & 2 & 2 & & 1 \\
\hline \multicolumn{2}{|c|}{ Frecuencia absoluta } & 59.37 & 50 & 28.12 & 12.5 \\
\hline \multicolumn{2}{|c|}{ Importancia total } & 64 & 48 & 38 & 5 \\
\hline
\end{tabular}

encontraron una gran abundancia de individuos de la familia Chydoridae dentro de las macrófitas, reforzando que su preferencia hacia dicho hábitat puede estar relacionada con la gran cantidad de detritus que origina la descomposición de estas plantas, que se constituye en una gran disponibilidad de materia orgánica. A pesar de que en diversidad dominaron las formas de hábitos litorales, la densidad estuvo dominada por Moina minuta, especie planctónica, endémica de la región Neotropical, de amplia distribución y hallada frecuentemente en este tipo de sistemas (Gavilán-Díaz 2000).

Las formas adultas de copépodos fueron poco abundantes, lo que de acuerdo con Allan (1976) obedece a que estos organismos son 
muy sensibles al estrés ambiental y tienen un desarrollo lento. La representación de los copépodos por parte del género Notodiaptomus ha sido frecuentemente hallada en otros sistemas tropicales (González 2007) y en el complejo cenagoso de Ayapel (Palacio 2008; Gallo-Sánchez et al. 2009), lo que obedece al endemismo neotropical de Notodiaptomus. Thermocyclops decipiens y Mesocyclops venezolanus Dussart forman una de las asociaciones más comunes dentro de los sistemas tropicales (Sendacz et al. 1985).

En estudios previos sobre la estructura del zooplancton en el complejo cenagoso de Ayapel no se hallaron diferencias entre sitios ubicados en la zona limnética del cuerpo de agua principal (Aguirre et al. 2005), ni entre sitios ubicados sobre el eje central del complejo cenagoso y sitios ubicados en la boca de los caños que alimentan el cuerpo de agua (GalloSánchez et al. 2009). Así mismo, en las ciénagas satélites al cuerpo principal, el número de individuos de zooplancton no presentó diferencias espaciales (Palacio 2008). En el presente estudio, sí se hallaron diferencias entre los dos hábitats en cuanto a composición, riqueza y diversidad (Eichhornia azurea). Lansac-Tôha et al. (2003) argumentan que la presencia de la vegetación es fundamental para el incremento de la riqueza zooplanctónica en un ecosistema, ya que en ellas se incrementa la relación superficie/volumen, lo que se traduce en una mayor disponibilidad de micro-hábitats y una fina partición espacial, con una consecuente baja competencia interespecífica, favoreciendo la presencia de organismos de hábitos planctónicos y bentónicos.

Las densidades no tuvieron un patrón espacial muy claro y sólo presentaron algunas tendencias que variaron con el tiempo; lo cual se reflejó en las altas equidades halladas en los dos hábitats, gracias a la codominancia de taxones como los nauplios, los copepoditos, Brachionus calyciflorus, Brachionus caudatus y Moina minuta, entre otros. Estudios como los de Jeppesen et al. (1997, 1998) y Genkai-Kato (2007), postulan que las macrófitas actúan como refugio para el zooplancton, y que por tanto el zooplancton es más numeroso y diverso en la proximidad a las plantas. Sin embargo, esto no es generalizable para todos los cuerpos de agua ni para todos los tipos de macrófitas, puesto que de acuerdo a Meerhoff (2006), a diferencia de lo ocurrido en la vegetación sumergida, que sí puede llegar a ser un refugio para el zooplancton de gran tamaño, la vegetación flotante de gran porte parece no producir este efecto y por el contrario, puede llegar a ser perjudicial para crustáceos de gran tamaño.

En este trabajo, la presencia de las plantas no favoreció una tendencia definida de mayor o menor densidad del zooplancton dentro de los parches de macrófitas conformados principalmente por las flotantes libres Eichhornia crassipes y Eichhornia heterosperma. Lo anterior estaría de acuerdo con la hipótesis planteada por Jeppesen et al. (1998), que establece que sólo en lagos eutróficos (que no es el caso del complejo cenagoso de Ayapel), las plantas acuáticas son un refugio importante para el zooplancton. Además de esto, se considera que la ausencia de un patrón espacial constante de la densidad obedece a la dinámica creada por el pulso hidrológico que reduce la predictibilidad en el establecimiento permanente de hábitats (Wantzen et al. 2008) y a la mezcla constante que caracteriza a este tipo de ecosistemas denominados "lagos someros".

No obstante, se debe resaltar que dentro de las plantas, las poblaciones de algunos individuos de hábitos bentónicos fueron más numerosas y que durante la inundación el zooplancton, y en especial los cladóceros, fueron menos numerosos en las aguas abiertas debido posiblemente a la interferencia mecánica que causan los sólidos entrantes (VillabonaGonzález 2010). Lansac-Tôha et al. (2003) encontraron que los cladóceros comúnmente sufren una disminución en las aguas abiertas durante la inundación, cuando la concentración de partículas suspendidas es alta. De esta manera, es probable que durante dicha temporada las macrófitas se constituyeran en una protección para estos organismos de hábitos filtradores (Paggi \& José de Paggi 2009), posibilidad que estos no tienen en aguas abiertas. 
Es de resaltar igualmente, que en la proximidad a la macrófita enraizada de hojas emergentes Eichornia azurea hubo una gran diversidad de formas litorales y una mayor densidad de taxones de hábitos bentónicos como Macrothrix sp., Lecane leontina y Alona sp.2. Lansac-Tôha et al. (2003) encontraron una mayor riqueza de cladóceros dentro de $E$. $a z u$ rea, debido principalmente a la contribución de las familias Chydoridae y Macrothricidae. Estos autores hallaron también una relación positiva entre la presencia de esta planta y la riqueza y densidad de rotíferos, influenciadas principalmente por especies de la familia Lecanidae.

A pesar de que la variación de la densidad zooplanctónica en la escala temporal no fue significativa estadísticamente, sí presentó una correlación negativa con el nivel del agua. Cuando los sistemas de inundación reducen su nivel de agua, es esperable un incremento en el número de organismos por unidad de volumen (González 2007). Carvalho (1983) explica que el incremento en la densidad zooplanctónica durante este periodo obedece a un enriquecimiento del cuerpo de agua, que consecuentemente trae consigo una mayor disponibilidad de alimento para el zooplancton.

Por otro lado, cuando ingresaron los tributarios al complejo cenagoso, la densidad y diversidad del zooplancton disminuyeron drásticamente en los sitios que tienen una influencia directa del río San Jorge (sitios ubicados al norte del complejo cenagoso y cercano al Caño Grande). Durante estos periodos y principalmente en dichos sitios, se hallaron larvas de peces Characiformes en las muestras de zooplancton, acontecimiento que anteriormente no había sido registrado. Wantzen et al. (2002) encontraron que con el incremento del nivel del agua, muchas especies de peces ingresan rápidamente desde el río a la zona de inundación, con el fin de hallar recursos alimenticios y lugares adecuados para su reproducción; por lo que es probable que exista un efecto de depredación. Además de lo anterior, el incremento particular en la densidad de algunos taxones durante determinados niveles muestra diferencias en el tiempo que reflejan la influencia que tiene el pulso de inundación sobre la estructura de poblaciones particulares del zooplancton (Carvalho 1983).

La relación inversa entre Conochilus dossuarius y los sólidos suspendidos, probablemente obedece a un fenómeno de interferencia en su mecanismo de filtración, ya que éste no posee la capacidad de discriminar entre las partículas vivas o inertes (Starkweather \& Bogdan 1980). Hamilton et al. (1990) encontraron que al igual que en el presente estudio, en el lago Tineo el rotífero Lecane proiecta fue más abundante en los periodos donde la columna de agua presentó altas concentraciones de oxígeno disuelto, mientras que Bonecker \& Lansac-Tôha (1996) hallaron una fuerte asociación entre la densidad de rotíferos y la disponibilidad de oxígeno disuelto y alimento (fitoplancton), lo que pudiera explicar la alta densidad zooplanctónica, influenciada principalmente por rotíferos, que se halló en la Miel, donde predominaron condiciones de alta oxigenación y alta biomasa fitoplanctónica (Villabona-González 2010.).

En conclusión, los resultados de este estudio revelan que las macrófitas favorecen una mayor riqueza zooplanctónica, constituida en gran medida por especies no planctónicas, ya que dichas plantas propician una mayor disponibilidad de micro-hábitats y una diversidad de ítems alimenticios. A pesar de que las macrófitas, en especial las de hojas flotantes libres (Eichhornia crassipes y E. heterosperma) no determinaron un patrón espacial constante en la densidad zooplanctónica, sí se evidenciaron tendencias particulares condicionadas a variaciones ecológicas creadas por el pulso de inundación. Adicionalmente, la macrófita enraizada de hojas emergentes Eichhornia azurea, contribuyó significativamente a una mayor diversidad y a la mayor densidad de taxones bentónicos.

Las fluctuaciones de las condiciones ambientales sólo se asociaron con cambios en la densidad de determinadas poblaciones y grupos del zooplancton, por lo que una gran proporción de individuos probablemente se encuentra adaptada al disturbio producido por 
el pulso de inundación y/o su comportamiento está asociado a otras variables no cuantificadas en el presente estudio, como el bacterioplancton y la presión de depredación por parte de peces e invertebrados.

\section{AGRADECIMIENTOS}

Este estudio fue financiado por COLCIENCIAS y la Sociedad Internacional de Limnología (SIL) a través de la fundación Tonolli. Agradecemos a CORPOAYAPEL y a los grupos GAIA y LimnoBasE de la Universidad de Antioquia por el apoyo logístico y económico. Agradecemos a Juan César Paggi y Susana José de Paggi por su colaboración en la determinación taxonómica y en la nomenclatura y distribución de las especies y a Castor Guisande, Nelson Aranguren, Claudia Bonecker, Mariana Meerhoff y Marlon Peláez por sus valiosos aportes al estudio.

\section{RESUMEN}

La mayoría de estudios ecológicos del zooplancton se han realizado en aguas abiertas. No obstante, es importante ampliar la exploración hacia hábitats como el de las macrófitas. En este estudio se evaluó la variación espacio-temporal de la estructura del ensamble de rotíferos y microcrustáceos asociados a macrófitas y se comparó con la variación de su estructura en aguas abiertas, para lo cual se tomaron muestras integradas de zooplancton y biomasa de fitoplancton usando una botella Schindler de $5 \mathrm{~L}$ en cuatro sitios de aguas abiertas y en cuatro sitios cubiertos por macrófitas durante diferentes niveles limnimétricos en el complejo cenagoso de Ayapel (Córdoba, Colombia). Las diferencias significativas de la estructura se evaluaron mediante Kruskal \& Wallis y discriminantes; y la similitud entre sitios de muestreo mediante Bray \& Curtis. Las macrófitas favorecieron la riqueza zooplanctónica; sin embargo, no hubo un patrón espacial constante en la densidad, pero sí tendencias particulares condicionadas por el pulso de inundación. La presencia de Eichhornia azurea (Pontederiaceae) contribuyó significativamente a la mayor diversidad y densidad de taxones bentónicos y sólo la densidad de algunos taxones y grupos del zooplancton se relacionó con las condiciones ambientales y la biomasa de fitoplancton.

Palabras clave: zooplancton, planos de inundación, macrófitas acuáticas, rotíferos, cladóceros, copépodos, bentos.

\section{REFERENCIAS}

Aguirre, N.J., J.A. Palacio, M.T. Fórez, A. Wills, O. Caicedo, L.F. Jiménez, N.E. Villegas, H. Grajales \& C. Palacio. 2005. Informe final del proyecto COLCIENCIAS “Análisis de la relación río-ciénaga y su efecto sobre la producción pesquera en el Sistema Cenagoso de Ayapel, Colombia”. Facultad de Ingeniería, Universidad de Antioquia, Medellín, Colombia.

Allan, J.D. 1976. Life history patterns in zooplankton. Am. Nat. 110: 165-180.

Alonso, M. 1996. Crustacea, Branchiopoda, p. 1-486. In M.A. Ramos, J. Alba, X. Bellés, J. Gonsálbes, A. Guerra, E. Macpherson, F. Martin \& J. Serrano Templado (eds.). Fauna Ibérica. Museo Nacional de Ciencias Naturales-CSIC, Madrid, España.

Andrade, C.E. 2001. Efecto de la fluctuación del nivel del agua sobre la estructura de la comunidad de rotíferos planctónicos en el lago Yahuarcaca. (Río AmazonasColombia). Tesis de Maestría, Universidad de los Andes, Bogotá, Colombia.

Aranguren, N. 1998. Estudio de los copépodos planctónicos (Crustacea) de la Ciénaga de Guarinosito, Magdalena Medio. Tesis de Maestría, Universidad de Los Andes, Bogotá, Colombia.

Bonecker, C. \& F.A. Lansac-Tôha. 1996. Community structure of rotifers in two environment of the upper River Paraná floodplain (MS)-Brazil. Hydrobiologia 325: $137-150$.

Bonecker, C., F.A. Lansac-Tôha \& D.C. Rossa. 1998. Planktonic and non planktonic rotifers in two environments of the upper Paraná river floodplain-MS, Brazil. Braz. Arch. Biol. Tech. 41: 447-456.

Carvalho, M. 1983. Efeitos da fluctuação do nível da água sobre a densidade e composição do zooplâncton em um lago de várzea da Amazonia, Brasil. Acta Amazonica $13: 715-724$.

Clesceri, L.S., A.E. Greenberg \& A.D. Eaton. 1998. Standard Methods for the Examination of Water and Wastewater. American Public Health Association (APHA), AWWA, WEF, Washington, EEUU.

Domínguez, A. 2001. Evaluación de la depuración de las aguas residuales provenientes de un sistema de tratamiento combinado de laguna de estabilización y laguna con jacinto de agua. Actual. Biol. 23: 75-82.

El Moor-Loureiro, L.M. 1997. Manual de identificação de cladóceros límnicos do Brasil. Universa-UCB, Brasilia, Brasil. 
Elías, M.E., E. Suárez-Morales, M. Gutiérrez-Aguirre, M. Silva-Briano, J. Granados Ramírez \& T. GarfiasEspejo. 2008. Cladocera y Copepoda de las aguas continentales de México. Guía ilustrada. Universidad Nacional Autónoma de México, Ciudad de México, México.

Estrada-Posada, A. 2007. Variación de la estructura y la distribución del zooplancton en función de los gradientes longitudinales en el embalse Río Grande II, Antioquia Colombia. Tesis de Doctorado, Universidad De Antioquia, Medellín, Colombia.

Fernando, C.H. 2002. A guide to tropical freshwater zooplankton: identification, ecology and impact on fisheries. Backhuys, Leiden, Holland.

Gallo-Sánchez, L., N. Aguirre-Ramírez, J. Palacio-Baena \& J.J. Ramírez-Restrepo. 2009. Zooplancton (Rotífera y Microcrustácea) y su relación con los cambios del nivel del agua en la ciénaga de Ayapel, Córdoba, Colombia. Caldasia 31: 327-341.

Gavilán-Díaz, R.A. 2000. Limnología comparativa de três lagoas neotropicais da bacia do Magdalena medio santandereano (Colombia), com ênfase no estudo da diversidade da comunidade zooplanctônica e sua relação com a dinâmica hidrológica do sistema regional. Tese de Doutorado, Universidade Federal de São Carlos, São Carlos, Brasil.

Gaviria, S. 2000. Guía de laboratorio para identificación de Cladóceros, Copépodos y Rotíferos. Curso Sistemática del Zooplancton de las aguas continentales de Colombia. Universidad de Antioquia, Instituto de Biología, Programa de Postgrado, Medellín, Colombia.

Gaviria, S. \& N. Aranguren. 2007. Especies de vida libre de la subclase Copepoda (Arthropoda, Crustacea) en aguas continentales de Colombia. Biota Colombiana 8: 53-68.

Genkai-Kato, M. 2007. Macrophyte refuges, prey behaviour and trophic interactions: consequences for lake water clarity. Ecol. Lett. 10: 105-114.

González, A.E. 2007. Influencia de Utricularia foliosa sobre la diversidad zooplanctónica en las dimensiones longitudinal y temporal de la Quebrada Yahuarcaca (Amazonia colombiana). Trabajo de grado, Universidad Distrital Francisco José de Caldas, Bogotá, Colombia.

Hamilton, S., S. Sippel, W. Lewis \& J.F. Saunders. 1990. Zooplankton abundance and evidence for its reduction by macrophyte mats in two Orinoco floodplain lakes. J. Plankton Res. 12: 345-363.
Inger, D., J. Deluque, S. Reyes \& T. Sierra. 2004. Composición de la comunidad de macroinvertebrados acuáticos asociados a las macrófitas de la Ciénaga del cerro San Antonio. VI Seminario Colombiano de Limnología, Montería, Colombia.

Jeppesen, E., T. Lauridsen, S.F. Mitchell \& C.W. Burns. 1997. Do planktivorous fish structure the zooplankton communities in New Zealand lakes? New Zeal. J. Mar. Freshwat. Res. 31: 163-173.

Jeppesen, E., T.L. Lauridsen, T. Kairesalo \& M. Perrow. 1998. Impact of submerged macrophytes on fishzooplankton relationships in lakes, p. 91-115. In E. Jeppesen, Ma. Søndergaard, Mo. Søndergaard \& K. Christoffersen (eds.). The structuring role of submerged macrophytes in lakes. Ecol. Stud. 131.

Koste, W. 1978. Rotatoria. Die radertiere Mitteleuropas. Überordnung Monogononta. Gebruder Borntrager, Berlin, Alemania.

Koste, W. \& R.J. Shield. 1987. Rotifera from Australian Inland Waters. II Epiphanidae and Brachionidae (Rotifera: Monogononta). Invertebrate Taxonomy 7: 949-1021.

Lansac-Tôha, F., L.F.M. Velho \& C. Bonecker. 2003. Influencia de macrófitas aquáticas sobre a estructura da comunidade zooplanctónica, p. 231-242. In S.M. Thomaz \& L.M Bini (eds.). Manejo de Macrófitas Aquáticas. Ecología e. EDUEM, Maringá, Brasil.

Meerhoff, M. 2006. The structuring role of macrophytes on trophic dynamics of shallow lakes under a climate warming scenario. Ph.D. Thesis, Aarhus Universitet, Dinamarca.

Paggi, J.C. 1995. Crustacea Cladocera, p. 909-951. In E. Lopretto \& G. Tell (eds.). Ecosistemas de aguas continentales: Metodología para su estudio. Ediciones Sur, La Plata, Argentina.

Paggi, J.C. \& S. José De Paggi. 2009. Curso Internacional Zooplancton Neotropical: Taxonomía, Ecología e Indicación Ambiental. Popayán, octubre 2-5.

Palacio, H. 2008. Distribución del zooplancton en cuatro ciénagas del sistema cenagoso de Ayapel, CórdobaColombia. Trabajo de grado, Universidad de Antioquia, Medellín, Colombia.

Pielou, E.C. 1969. An introduction to mathematical ecology. Wiley, Nueva York, EEUU.

Reid, J.W. 1985. Chave de identificação e lista de referências bibliográficas para as espécies continentais suramericanas de vida livre da ordem Cyclopoida 
(Crustacea, Copepoda). Bolm. Zool. São Paulo. 9: 17-143.

Rossa, D.C., F. Lansac-Tôha, C. Bonecker \& L. Velho. 2001. Abundance of cladocerans in the littoral regions of two environments of the upper Paraná river floodplain, Mato Grosso do Sul, Brazil. Rev. Bras. Biol. 61: 45-53.

Rossa, D.C. \& C. Bonecker. 2003. Abundance of planktonic and non-planktonic rotifers in floodplain lakes of the Upper Paraná River floodplain. Amazoniana 17: 567-581.

Sartory, D.P. \& J.U. Grobbelaar. 1984. Extraction of chlorophyll $a$ from freshwater phytoplankton for spectrophotometric analysis. Hydrobiologia 114: 177-187.

Segers, H. 1995. Rotifera. Volume 2: The Lecanidae (Monogonota). Guides to the identification of the microinvertebrates of the continental waters of the world 6. SPB, La Haya, Holanda.

Sendacz, S. \& E. Kubo. 1982. Copepoda (Calanoida e Cyclopoida) de reservatórios do Estado de São Paulo. B. Inst. Pesca. 9: 51-89.

Sendacz, S., E. Kubo \& M. Cestarolli. 1985. Limnologia de reservatorios do sudeste do estado de São Paulo. B. Inst. Pesca 9: 178-189.

Shannon, C.E. \& W. Weaver. 1949. The mathematical theory of communication. University of Illinois, Illinois, Estados Unidos.

Shield, R.J. \& W. Koste. 1992. Rotifera from Australian inland waters VIII. Trichocercidae (Monogononta). Trans. Roy. Soc. S. Aust. 116: 1-27.

Shield, R.J. \& W. Koste. 1993. Rotifera from Australian inland waters. IX. Gastropodidae, Synchaetidae, Asplanchnidae (Rotifera: Monogononta). Trans. Roy. Soc. S. Aust. 117: 111-139.

Simpson, E.H. 1949. Measurement of diversity. Nature 163: 688 .
Sladecek, V. 1983. Rotifers as indicators of water quality. Hydrobiologia 100: 169-171.

Starkweather, P. \& K. Bogdan. 1980. Detrital feeding in natural zooplankton communities discrimination between live and death algal foods. Hydrobiologia 73: 83-85.

Suárez Morales, E., J. Reid, T. Lliffe \& F. Fiers. 1996. Catálogo de los copépodos (Crustácea) continentales de la península de Yucatán, México. Colegio de la frontera sur, Chatumal, México.

Thomaz, S., M.L.M. Bini \& T.A. Pagioro. 2004. Métodos em limnologia: macrófitas aquáticas, p. 193-212. In C. Bicudo \& D.C. Bicudo (eds.). Amostragem em limnologia. São Carlos, Brasil.

Villabona-González, S. 2010. Comparación de la estructura de rotíferos y microcrustáceos en dos hábitats: macrófitas y aguas abiertas, en diferentes periodos limnimétricos en el complejo cenagoso de Ayapel, Córdoba, Colombia. Tesis de Maestría, Universidad de Antioquia, Medellín, Colombia.

Wallace, R.L. \& W.T. Edmonson. 1986. Mechanism and adaptative significance of substrate selection by a sessile rotifer. Ecology 67: 314-323.

Wantzen, K.M., F.A. Machado, M. Voss, H. Boriss \& W.J. Junk. 2002. Floodpulse-induced isotopic changes in fish of the Pantanal wetland. Braz. Aquat. Sci. 64: 251.

Wantzen, K., W. Junk \& K. Rothhaupt. 2008. An extension of the floodpulse concept (FPC) for lakes. Hydrobiologia 613: 151-170.

Wills, A. \& G.A. Gómez. 2008. Caracterización del hidroperiodo en la ciénaga de Ayapel, p. 152-154. In M.T. Florez, J.O. Rangel-Ch \& L.N. Parra-S. (eds.). Libro de resúmenes Primer Congreso Nacional Ciénagas y Lagunas de Colombia. Homenaje al prof. Dr. Thomas van der Hammen. Universidad de Antioquia, Universidad Nacional de Colombia, Medellín-Bogotá, Colombia. 